\title{
Lidil
}

Revue de linguistique et de didactique des langues

\section{Langues maternelles : contact, variations et enseignement. Le cas de la langue amazighe}

RisPAIL Marielle (dir.), 2005, en collab. avec Nora Tigziri, L'Harmattan, «Espaces discursifs », 340 p.

\section{Myriam Abouzaïd}

\section{(2) OpenEdition}

\section{Journals}

Édition électronique

URL : http://journals.openedition.org/lidil/74

DOI : $10.4000 /$ lidil.74

ISSN : 1960-6052

Éditeur

UGA Éditions/Université Grenoble Alpes

\section{Édition imprimée}

Date de publication : 1 juin 2006

ISBN : 2-914176-14-7

ISSN : 1146-6480

\section{Référence électronique}

Myriam Abouzaïd, «Langues maternelles : contact, variations et enseignement. Le cas de la langue amazighe », Lidil [En ligne], 33 | 2006, mis en ligne le 01 octobre 2007, consulté le 21 septembre 2020 URL : http://journals.openedition.org/lidil/74 ; DOI : https://doi.org/10.4000/lidil.74

Ce document a été généré automatiquement le 21 septembre 2020.

(c) Lidil 


\section{Langues maternelles : contact, variations et enseignement. Le cas de la langue amazighe}

RisPAIL Marielle (dir.), 2005, en collab. avec Nora Tigziri, L'Harmattan, « Espaces discursifs », 340 p.

Myriam Abouzaïd

1 Cet ouvrage collectif est composé de 28 contributions, dont environ la moitié ont été présentés lors du Colloque de Tizi Ouzou (Algérie) de 2003 intitulé « L'enseignement des langues maternelles». Marielle Rispail, didacticienne et sociolinguiste, réunit ici un vaste ensemble de propos autour de la langue amazighe (berbère). Au moment où, au Maroc et en Algérie, les institutions s'engagent véritablement dans l'enseignement de cette langue jusqu'alors minorée, il semble, en effet, pertinent de faire le bilan des étapes franchies et de préciser le chemin qu'il reste encore à parcourir.

La démarche de l'auteur ambitionne toutefois de dépasser le cadre de l'amazighe et permet d'aborder la question plus générale de l'enseignement des langues minorées en situation de contacts de langues. Le titre de l'ouvrage laisse entrevoir la grande variété des thèmes traités; mais c'est également la diversité des approches retenues par les différents auteurs qui caractérise ce recueil. Le lecteur y trouvera aussi bien des analyses critiques de programmes d'enseignement, des propositions d'activités pédagogiques que des essais théoriques, des synthèses de projet d'aménagement linguistique et des comptes-rendus d'enquête sociolinguistique.

3 Ces contributions sont organisées en quatre grandes parties, et chacune d'elles constitue un chapitre. La première partie traite de questions essentiellement théoriques où les différents auteurs analysent des notions de base qui méritent d'être clairement (re)définies par souci de rigueur scientifique. La deuxième partie intitulée «Éclairages extérieurs » offre une mise en perspective de la question de l'enseignement des langues minorées ou immigrées, à travers des analyses de contextes distincts du berbère. Les deux dernières parties se recentrent sur la langue amazighe et permettent d'en dégager les spécificités. 
Dans sa partie introductive, Marielle Rispail anticipe les objections pouvant naitre du choix de l'usage de l'expression «langue maternelle » (LM) et suggère que " même si elle n'est pas satisfaisante du point de vue scientifique, elle est dynamique sur le plan intellectuel si on essaie d'en interroger les limites ». Certains auteurs reviennent sur ce terme souvent utilisé de façon imprécise, comme Dalila Morsly (I, chap. 4) qui souligne son caractère polysémique ainsi que son manque de pertinence dans certains contextes plurilingues où la LM se décline forcément au pluriel. On notera toutefois que pour d'autres auteurs (Dalila Arezki, III, chap. 2), la LM (au singulier) est bien celle de la mère et que cela en fait une langue "naturelle». Le débat terminologique reste donc ouvert... Marielle Rispail ajoute, dans son introduction, que «dire que le berbère est langue maternelle, et que cela doit guider son enseignement, gomme le paramètre principal de sa situation, à savoir qu'il a été dans la plupart des pays où il est parlé, une langue minorée ». Les contributions s'inscrivent ainsi dans deux dimensions étroitement liées, car « considérer le berbère comme langue maternelle signifie qu'on va privilégier la découverte des structures d'une langue qu'on parle déjà, et que priorité sera donnée aux règles explicites et à l'apprentissage de l'écrit " et «considérer le berbère comme une langue minorée suppose aussi qu'on mette l'accent [...] sur les façons de passer d'un oral marqué par la variation à un écrit assez standardisé pour être enseignable ».

Sur le plan de l'enseignement des LM, Morteza Mahmoudian revient sur ce que signifie « connaitre sa LM » ou la « conscience linguistique » en analysant les différents champs et niveaux de connaissance que l'on peut avoir de sa propre langue. Il souligne ainsi l'apport réciproque se dégageant de l'interaction entre conscience linguistique et pratique pédagogique (I, chap. 2). Mohand-Akli Haddadou présente les résultats d'une enquête visant à mesurer les connaissances lexicales d'enfants berbérophones à leur entrée à l'école et propose des pistes didactiques pour un enseignement du lexique en LM (III, chap.7).

6 Sur le thème de l'enseignement des langues minorées, Hakim Smaïl propose une réflexion critique sur l'évolution de l'enseignement du berbère au Maroc et en Algérie. Il insiste sur la diversité des situations sociolinguistiques selon les aires dialectales, sur le manque d'objectifs clairement définis, et sur le manque de rigueur des stratégies pédagogiques adoptées (s'agit-il de l'enseignement d'une L1, L2, LE ou un mélange des trois ?). L'auteur affirme également que l'enseignement de l'amazighe ne représente que la partie visible de l'iceberg, et qu'il ne peut suffire à assurer l'épanouissement et la pérennité de la langue (I, chap. 5). Ahmed Boukous présente, lui aussi, une synthèse critique des enjeux de la récente réforme dans la politique linguistique éducative du Maroc. Il suggère, entre autres, que l'introduction de l'amazighe dans la sphère scolaire peut contribuer à remédier à certains dysfonctionnements du système éducatif (III, chap. 1).

7 Itziar Idiazabal aborde les défis de l'enseignement du basque en Espagne et en France, à travers l'historique de cet enseignement et la gestion de la variation. Il propose ensuite une description détaillée des différents modèles d'enseignement bilingue ayant vu le jour dans ce contexte. Il termine sur cette idée forte que la priorité consiste à favoriser l'usage de la langue et rappelle ainsi que les projets de normativisation et de réglementation ne doivent pas occulter cette priorité (II, chap. 3). Bouteldja Riche et Sadia Belkhir proposent une analyse de l'enseignement du gallois au Pays de Galles qui pourrait servir de modèle à l'enseignement de l'amazighe en Algérie. Les auteurs, à 
travers une approche historique, soulignent l'importance d'analyser en profondeur la situation diglossique du pays concerné (II, chap. 4). Une incursion dans le contexte catalan permet à Joaquim Dolz de répertorier certaines conditions sociales, économiques et politiques favorisant l'enseignement d'une langue minorée (I, chap. 6).

8 Enfin, Zahir Meksem, à travers une enquête sociolinguistique, étudie les représentations de jeunes apprenants d'amazighe dans la ville de Bejaïa. A partir de la réception des écrits urbains, l'auteur montre l'impact de la présence de l'amazighe dans la sphère scolaire sur ces représentations (III, chap. 5).

La problématique de l'impact psychologique de la minoration des langues premières sur leurs locuteurs est abordée par Dalila Arezki dans un chapitre intitulé «Les enjeux de la LM sur le développement psychoaffectivocognitif de l'enfant» (III, chap. 2). Ahmed Boukous insiste sur la fonction structurante des LM, et sur l'acculturation, l'aliénation et les comportements langagiers schizoglossiques que peut entraîner leur rejet (IV, chap. 1). Manuela Caballero, quant à elle, étudie le rôle des cours d'espagnol ELCO (Enseignement des langues et cultures d'origine) dans la maîtrise de l'écrit, en Suisse romande. A partir d'une analyse comparée de productions écrites d'enfants migrants et non migrants, l'auteur démontre qu'un ancrage dans la LM apporte "maitrise linguistique et stabilité identitaire» (II, chap. 2). On peut néanmoins remarquer que, dans l'ensemble, des lacunes méthodologiques sont perceptibles dans le traitement de ce thème central: on aurait aimé pouvoir compter sur des démonstrations plus richement étayées afin de parvenir à des conclusions solides dans ce domaine.

10 La question de l'aménagement $d u$ corpus donne lieu à des confrontations d'opinion très fertiles. Ainsi, Salem Chaker rappelle que l'on ne peut faire l'impasse sur la question épineuse de la définition du standard de langue : « une ou des langues berbères? ». Il explique et justifie sa position, aujourd'hui célèbre dans la sphère berbérisante, qu'il nomme "standardisation convergente ». Ce compromis, qui se veut à la fois réaliste et progressiste, propose d'inscrire l'action de codification dans la durée. Abdallah Boumalk, à son tour, aborde le thème de la construction d'une norme en amazighe. Il nous fournit une synthèse claire et détaillée de la résolution de ce problème au Maroc. Présentant successivement les raisons du choix d'une «standardisation graduelle », la normalisation graphique et la normalisation lexicale, ce linguiste chercheur à l'Institut royal de la Culture Amazighe (IRCAM) explique les priorités du Centre de l'Aménagement linguistique et mentionne certains écueils à éviter (III, chap. 3). Aux côtés de ces contributions optimistes et tournées vers l'avenir, on trouve également des points de vue laissant poindre un certain scepticisme dans le domaine de la standardisation. Ainsi, Nora Tigziri et Amar Nabti, à travers une enquête auprès d'enseignants d'amazighe, mettent à l'épreuve le programme d'enseignement de l'amazighe en $7^{\mathrm{e}}$ année fondamentale en Algérie et offrent une réflexion enrichie de paroles d'enquêtés sur le risque d'aboutir à une « langue hermétique » qui « n'éveillera plus aucun souvenir auprès des élèves » (IV, chap. 3). Mehdi Kaddouri nous donne, lui aussi, un aperçu nuancé du travail de standardisation effectué au Maroc. Ce linguiste tarifitophone affirme en effet que « le système de l'IRCAM semble pénaliser la créativité et s'opposer à l'évolution qui est le propre de tout système linguistique » (IV, chap. 8).

11 En guise d'apport extérieur en matière de gestion de la variation, Joaquim Dolz aborde les thèmes de l'intercompréhension et de rejet des normes standard dans certaines régions de Catalogne (I, chap. 6). Rémi Jolivet, lui, propose une réflexion sur quelques aspects de la réforme de l'enseignement du français en Suisse romande. Il met en 
évidence le poids normatif de la variété dominante que constitue le français de France, malgré le désir affiché par les didacticiens locaux de partir des acquis des élèves. Ce qu'il considère comme une contradiction est accentué par la persistance de la supériorité de l'écrit sur l'oral dans les représentations sociales (II, chap. 1).

Ce couple oralité/écriture constitue précisément, nous semble-t-il, le dernier grand thème abordé dans cet ouvrage. Nadia Berdous étudie ainsi l'impact de la situation sociolinguistique de l'amazighe sur la compétence écrite de collégiens kabylophones en LM. Elle constate que "faute de bien connaître le code écrit en tamazight, ils transposent le code oral ou empruntent celui de l'arabe" (IV, chap. 5). Hassina Kherdouci, quant à elle, se propose d'exploiter l'oralité de l'amazighe à bon escient et invite, exemples à l'appui, à puiser dans les chansons féminines pour qui souhaite didactiser la langue. L'auteur suggère, à travers une approche socio-

littéraire du texte oral, d'agir d'une pierre deux coups : rendre à l'oralité un statut respectable et s'atteler au problème du statut de la femme (III, chap. 6). Plusieurs autres contributions (Abdelkader Bezzazi, III, chap. 7) évoquent la richesse du patrimoine oral amazighe et ouvrent des pistes intéressantes pour leur exploitation pédagogique. Marielle Rispail montre comment, de façon générale, les chansons "populaires » peuvent être utilisées dans l'enseignement. A travers une démonstration truffée d'exemples, elle fait passer la chanson en langue minorée du stade d'objet social à celui d'outil didactique par excellence (II, chap. 5).

Il est intéressant de remarquer que l'ouvrage s'achève sur la parole d'un berbérophone inquiet. En effet, Mehdi Kaddouri attire l'attention sur le fait que « la centration sur l'écrit, alors que la culture amazighe est fondamentalement orale, est une forme de résignation devant la poussée de l'uniformisation ». Il dénonce ainsi un " assujettissement de la parole, de l'oralité, fondatrice de la culture amazighe, à l'écrit » (IV, chap. 8).

Précisons que répartir les interventions en cinq grands thèmes, comme nous venons de le faire, s'avère relativement artificiel et restrictif. En effet, il existe une forte imbrication de ces domaines, et chaque auteur s'exprime nécessairement sur un ensemble de problématiques. C'est la raison pour laquelle le lecteur relèvera à la fois des récurrences et des contradictions d'un chapitre à l'autre. Loin de constituer un point faible, ceci souligne un des objectifs principaux de cet ouvrage: ouvrir le dialogue. Au final, il nous semble, d'une part, que la multiplicité des points de vue permet au lecteur déjà initié au domaine de l'amazighe de récolter de nombreux éléments de réflexion et de remettre en question certaines pseudo évidences. D'autre part, les éclairages extérieurs ainsi que la démarche générale visant à enrichir les pratiques pédagogiques par les apports de la recherche en sociolinguistique élargissent le débat et aboutissent ainsi à un ouvrage qui intéressera sans aucun doute un public non spécialiste du berbère. 


\section{AUTEUR}

MYRIAM ABOUZAÏD

Lidilem. 